\title{
Le séisme de Pisco du 15 août 2007 : entre urgence et reconstruction
}

Robert D'Ercole, Jérôme Chandes, Hugo Perfettini et Laurence Audin

\section{OpenEdition}

\section{Journals}

\section{Édition électronique}

URL : http://journals.openedition.org/echogeo/2109

DOI : 10.4000/echogeo.2109

ISSN : 1963-1197

\section{Éditeur}

Pôle de recherche pour l'organisation et la diffusion de l'information géographique (CNRS UMR 8586)

\section{Référence électronique}

Robert D'Ercole, Jérôme Chandes, Hugo Perfettini et Laurence Audin, « Le séisme de Pisco du 15 août 2007 : entre urgence et reconstruction », EchoGéo [En ligne], Sur le Vif, mis en ligne le 18 décembre 2007, consulté le 19 avril 2019. URL : http://journals.openedition.org/echogeo/2109; DOI : 10.4000/ echogeo.2109

Ce document a été généré automatiquement le 19 avril 2019.

\section{cc) $९$ (†)}

EchoGéo est mis à disposition selon les termes de la licence Creative Commons Attribution - Pas d'Utilisation Commerciale - Pas de Modification 4.0 International 


\title{
Le séisme de Pisco du 15 août 2007 : entre urgence et reconstruction
}

\author{
Robert D'Ercole, Jérôme Chandes, Hugo Perfettini et Laurence Audin
}

\section{Introduction}

1 Le séisme de Pisco qui a affecté la côte du Pérou central le 15 août 2007 présente des caractéristiques particulières ayant influencé ses conséquences humaines et matérielles : relativement peu de victimes et un très grand nombre de familles sinistrées en raison de l'étendue des dommages ayant affecté les habitations. L'objet de cet article vise à rendre compte de ces caractéristiques et des effets du séisme. En même temps, il place l'accent sur les aléas d'une gestion de crise difficile et sur une situation de crise qui perdure mêlant urgence, réhabilitation et reconstruction. L'article est écrit par quatre chercheurs représentant des disciplines différentes, ayant vécu le séisme et ses conséquences à Lima, puis dans les zones les plus affectées. Leur analyse relève de lectures (notamment de rapports et d'articles de presse) et surtout de nombreux entretiens menés auprès de victimes du séisme et d'acteurs de la gestion de crise.

\section{Les caractéristiques du séisme de Pisco}

2 Le séisme est survenu le 15 août 2007 à 18:40 heure locale. La localisation la plus précise de son épicentre le situe à $76.8520 \mathrm{~W}, 13.4914 \mathrm{~S}$, à une profondeur de $26.4 \mathrm{~km}$, à moins de $100 \mathrm{~km}$ au large de la cote péruvienne (Hernando Tavera, Institut Géophysique du Pérou, communication personnelle), a l'ouest de la ville de Pisco. 


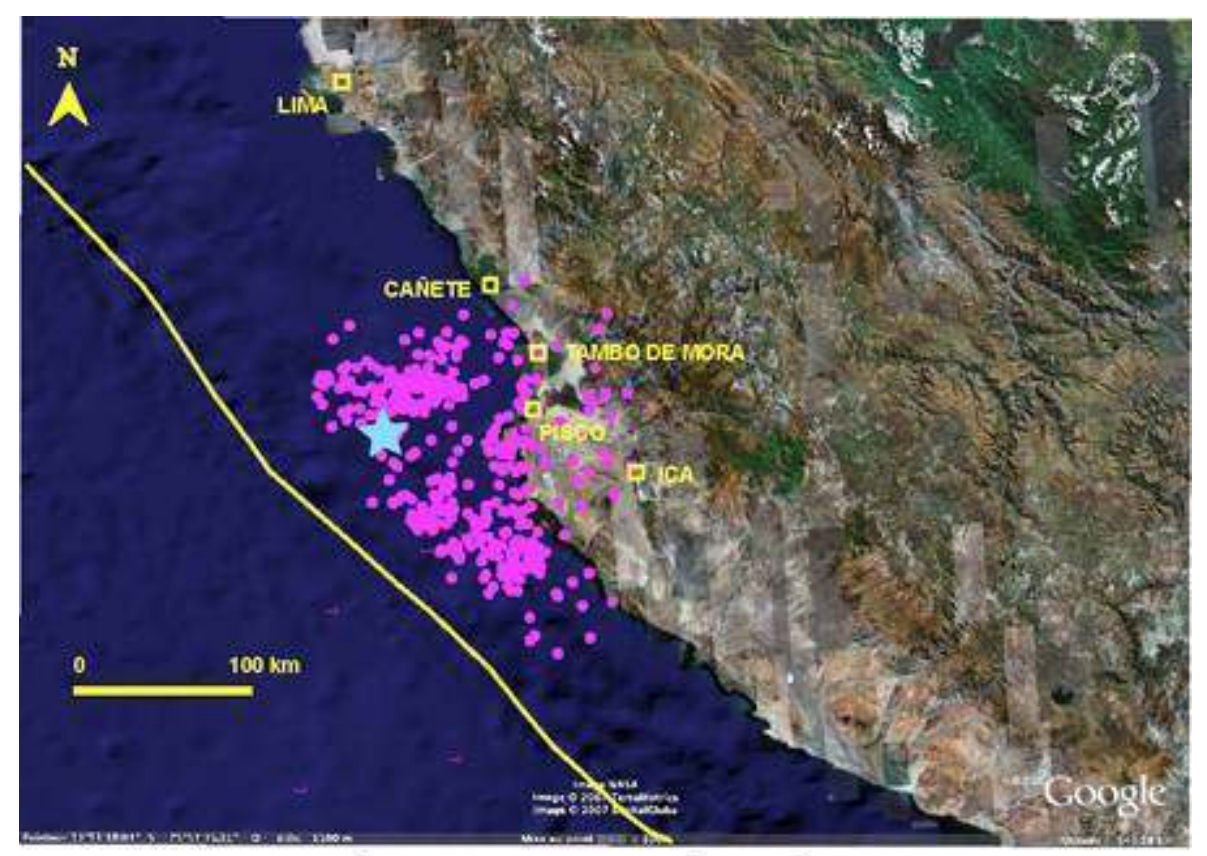

Figure 1 - Epicentre du séisme de Pisco du 15 août 2007 (étoile bleu ciel) et principales répliques des 15 premiers jours (cercles magenta).

Source: Hernando Tavera, Institut Geophysique du Pérou.

La ligne jaune correspond à la frontiére de la plaque de Nasca et de la plaque sud Américaine.

3 Sa magnitude Mw (magnitude de moment) varie entre 7.9 et 8.1 suivant les sources ${ }^{1}$. Le séisme s'est produit le long de la zone de subduction, interface correspondant au contact des plaques de Nasca avec la plaque Sud Américaine. Dans la région de Pisco, ces deux plaques ont une vitesse de convergence relative de plus de $7 \mathrm{~cm} / \mathrm{an}$, ce qui en fait une des zones les plus actives du globe sur le plan sismique.

Le séisme de Pisco est très particulier à plusieurs égards. Deux secousses ont été séparées par une période de 60 secondes, chacune d'entre elles correspondant individuellement a un séisme de $\mathrm{Mw}=7.5$. Entre ces deux secousses, les sismomètres installés au Pérou sont restes silencieux, ce qui a d'abord laissé penser à deux événements distincts. L'US Geological Survey, organisme en mesure de localiser les plus gros séismes du monde en un temps record (quelques minutes) a d'ailleurs proposé le même scénario. Il semblerait cependant que ces deux secousses n'en constituent qu'une seule. En effet, certains résultats préliminaires (interférométrie radar, distribution spatiale des répliques) suggèrent que le séisme ne s'est pas arrêté après la première rupture, et a continué à se propager a grande vitesse (de l'ordre de $2 \mathrm{~km} / \mathrm{s}$ ) sans rayonner d'ondes sismiques, ce qui explique le scénario initial envisagé. Ce mode de rupture très particulier a permis de limiter les dégâts dus aux ondes sismiques, ces dernières ayant "disparu" pendant près de 60 secondes. En revanche, ce glissement bien qu'asismique et n'affectant pas les habitations, a contribué à déplacer le fond marin de plusieurs mètres, ce qui a entraîné le développement d'un tsunami. Une vague de 2 a 3 mètres de hauteur a ainsi frappé le sud de la péninsule de Paracas, heureusement quasiment inhabitée. Trois victimes sont à reporter dans le site de Lagunilla, où la vague est localement montée à $6 \mathrm{~m}$, entraînant des embarcations à plus de $2 \mathrm{~km}$ à l'intérieur des terres. 
Photo 1 - Bateaux transportés sur plus de $3 \mathrm{~km}$ et cassés par le tsunami dans la baie de Lagunillas (sud de la péninsule de Paracas)

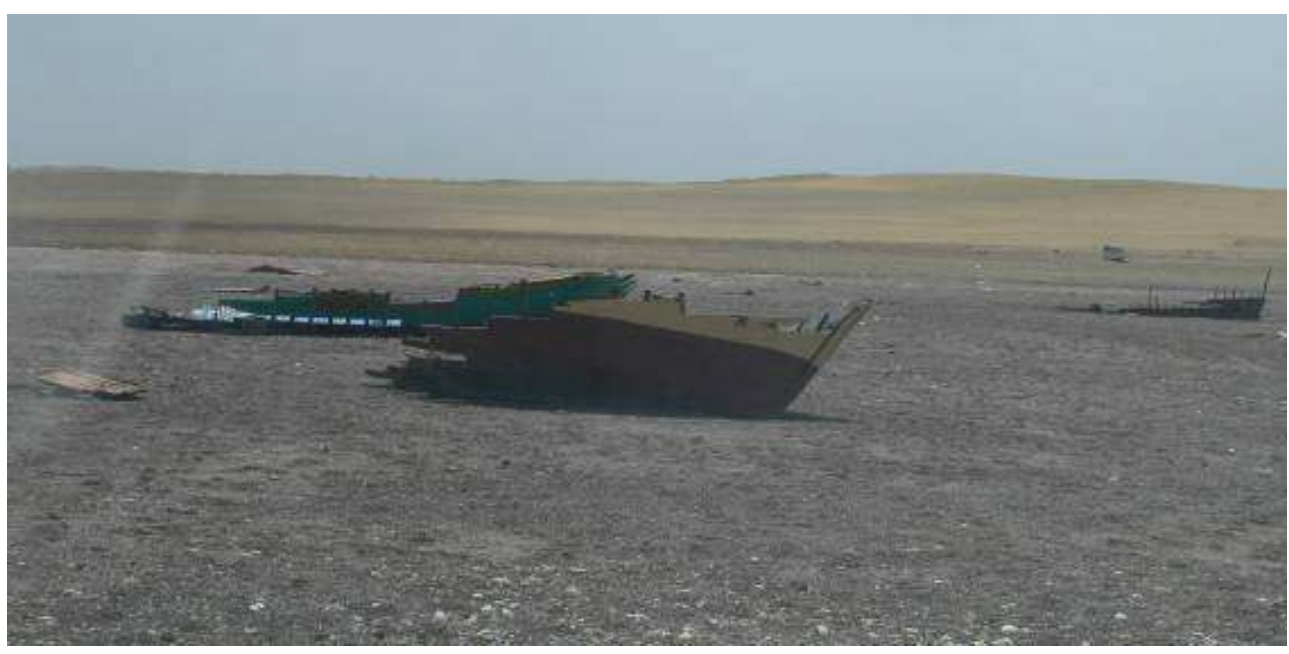

Auteur : Laurence Audin - Août 2007

Photo 2 - Destruction par le tsunami d'un site touristique de la pointe de la baie de Lagunillas

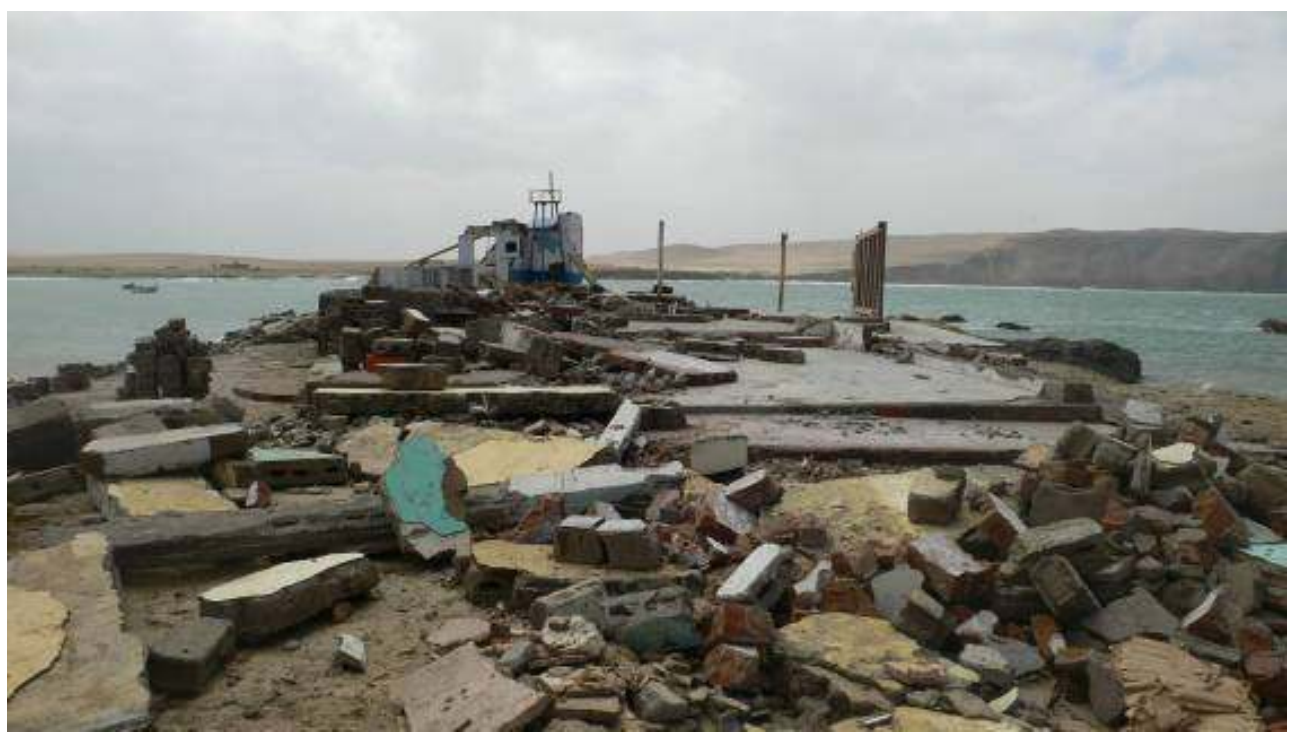

Auteur : Laurence Audin - Août 2007

Une autre caractéristique peu commune du séisme de Pisco est qu'il semble s'être propagé sous le continent, jusqu'à la ville de Ica. Dans la plupart des séismes de subduction, la cote correspond grossièrement à la base de la zone sismogène, et une telle propagation de la rupture dans le continent n'est normalement pas possible. La présence de la dorsale de Nasca, structure sous-marine orientée perpendiculairement a la cote, et qui s'élève de plus de $2 \mathrm{~km}$ au-dessus du niveau du plateau océanique, est, semble-t-il, responsable de la complexité de ce séisme. En subductant sous la plaque de Nasca, cette ride change le comportement frictionnel de la zone sismogène, et agit comme une sorte de savonnette. La partie asismique de la rupture pourrait par conséquent correspondre à la propagation de la rupture le long de la dorsale. Pour toutes les anomalies qu'il présente, ce séisme est donc exceptionnel. 


\section{Effets différentiels du séisme}

Les données officielles provenant de la protection civile péruvienne (INDECI, 29/10/2007) font état de 519 morts et 1291 blessés. Le nombre des victimes est faible par rapport aux dommages enregistrés: 92152 logements ont été entièrement détruits ou sont inhabitables, 44810 autres ont été endommagés. En revanche, plus de 430000 personnes ont occupé et, pour la majorité d'entre elles, occupent encore les abris provisoires distribués lors des opérations d'urgence (tentes, habitat en cannes tressées - esteras - ou en bois).

7 Le nombre relativement faible de victimes (très largement inférieur aux 67000 victimes du séisme du 15 mai 1970 qui affecta le nord du pays) s'explique par la conjonction de plusieurs facteurs :

- l'heure à laquelle le séisme s'est produit : à $18 \mathrm{~h} 40$ la plupart des personnes affectées n'étaient plus sur le lieu de leur travail (bureaux notamment), les établissements scolaires n'étaient plus occupés et la population n'a pas été surprise durant la nuit en plein sommeil ;

- les 60 secondes séparant les deux secousses ont permis à la population d'évacuer leurs habitations (pour la plupart des maisons de un à deux étages) avant que ces dernières ne s'effondrent ;

- l'absence de grosses répliques ; la plus grosse reportée (le 16 août 2007 à 0h17) était de $\mathrm{Mw}=6.3$, ce qui est anormalement bas pour un séisme de cette taille ; les répliques n'ont donc pas fortement alourdi le bilan des dégâts, ce qui est généralement le cas ;

- la distance relativement courte (moins de $200 \mathrm{~km}$ ) qui sépare les zones les plus affectées de la capitale du Pérou : en dépit de voies routières interrompues et des difficultés initiales de la gestion de crise, les secours ont pu être acheminés assez rapidement.

Du fait de la proximité de la zone de rupture, la majorité des victimes et des dégâts se situent près de la côte, dans les provinces de Chincha. Pisco et Ica. 


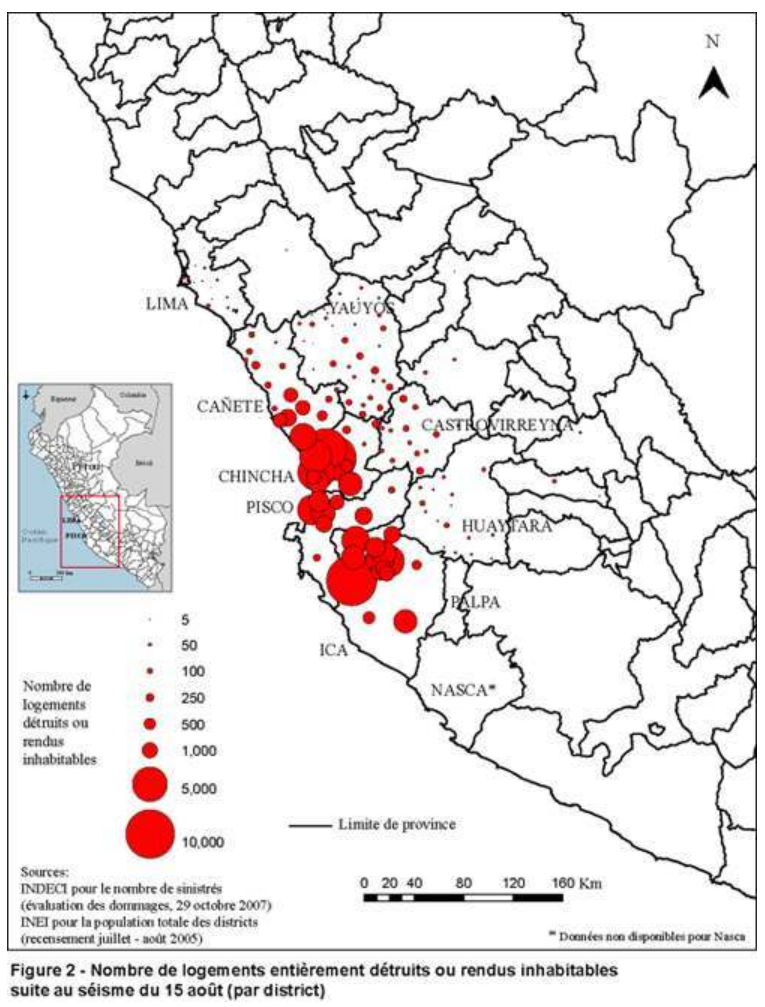

9 La province de Cañete a également été sérieusement affectée. A Lima / Callao, a $200 \mathrm{~km}$ de l'épicentre, les dommages sont loin d'être négligeables : 2 morts, 5 blessés, plus de 200 logements détruits et plusieurs centaines endommagés, essentiellement dans les secteurs les plus pauvres de l'agglomération. Le séisme a également été ressenti à l'intérieur du pays, sur les versants andins et même sur l'altiplano où le rapport population sinistrée/ population totale des districts est souvent très élevé.

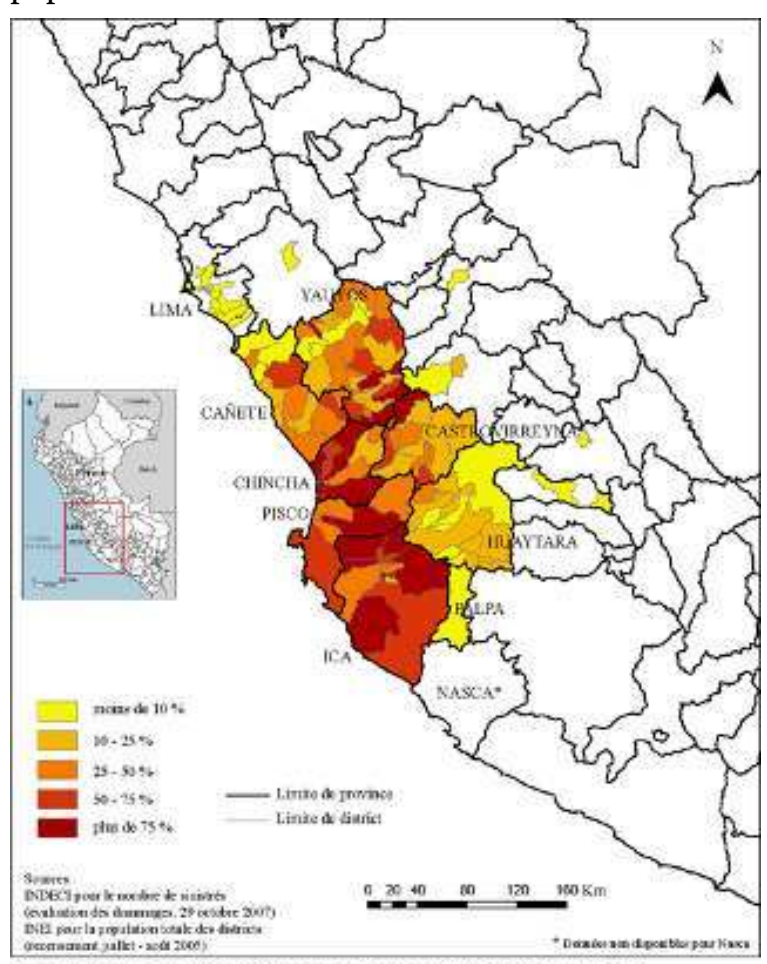

Figure 3- Pouroentage de population sinistrbe par rapport à la population totale

Figure 3. Fouroentage de population sin
suite au seisme du 15 aeout [par district) 
10 Le séisme a révélé la forte vulnérabilité des établissements scolaires (643 salles de classe détruites et 635 endommagées), des églises (notamment l'église San Clemente de la place centrale de Pisco où plus de 150 personnes sont décédées) et des établissements de santé (14 d'entre eux ont été détruits et 112 endommagés).

Photo 3 - Destruction de l'église en adobe et en bois de Chincha Baja.

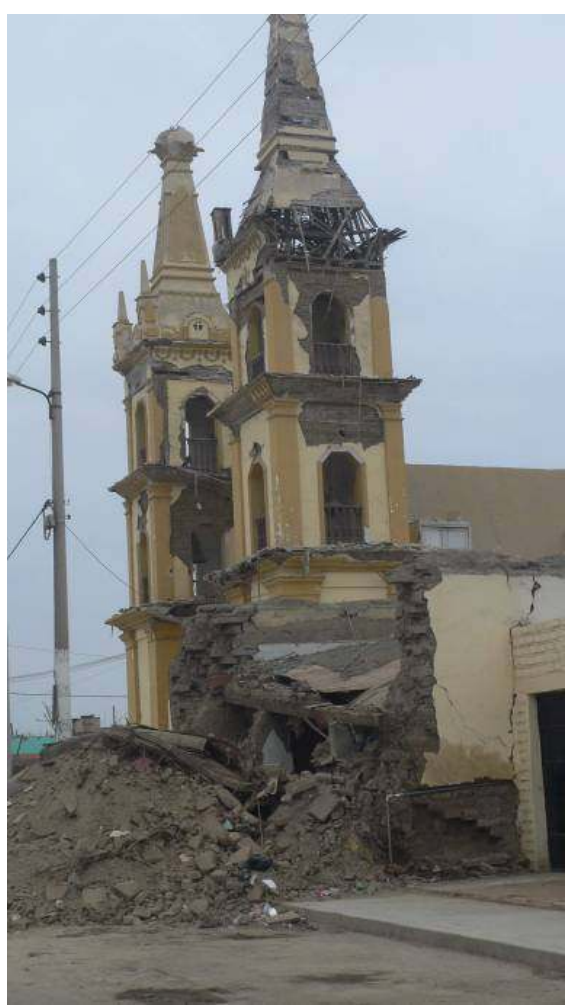

Auteur : Laurence Audin - Août 2007 
Photo 4 - Destruction partielle de I'hôpital Rene Toche Groppo (Chincha Baja)

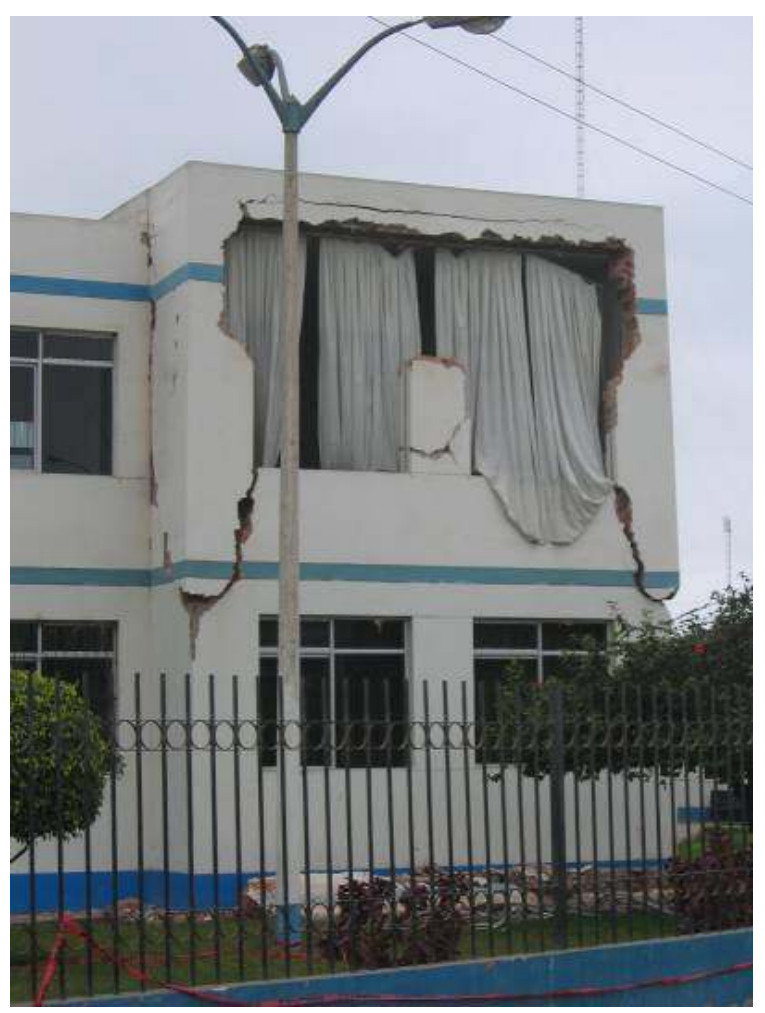

Auteur : Robert D'Ercole - Août 2007

243 personnes ont trouvé la mort dans les établissements de santé et près de 600 y ont été blessées ${ }^{2}$ (soit près de la moitié du nombre total de victimes, tuées ou blessées).

Le coût économique du séisme est également élevé. La région de Ica est l'un des plus importants centres agricoles du pays (fruits, légumes, vigne) et de nombreuses activités gravitent autour de la pêche et du tourisme. De nombreuses infrastructures hôtelières ont été détruites ou fortement endommagées, de même que des sites touristiques particulièrement fréquentés comme le site de la "cathédrale ", pont naturel constitué par l'érosion marine, qui s'est effondré suite au séisme. Même si les infrastructures agricoles et industrielles ont été peu affectées (Taucer et al., 2007), les conséquences économiques, non encore évaluées précisément, sont élevées. Elles sont liées, en particulier, à l'interruption ou au fort ralentissement des activités durant plusieurs semaines (employés mobilisés par les opérations d'urgence, à titre personnel ou collectif). 
Photo 5 - Destruction d'une maison en adobe à Chincha Alta

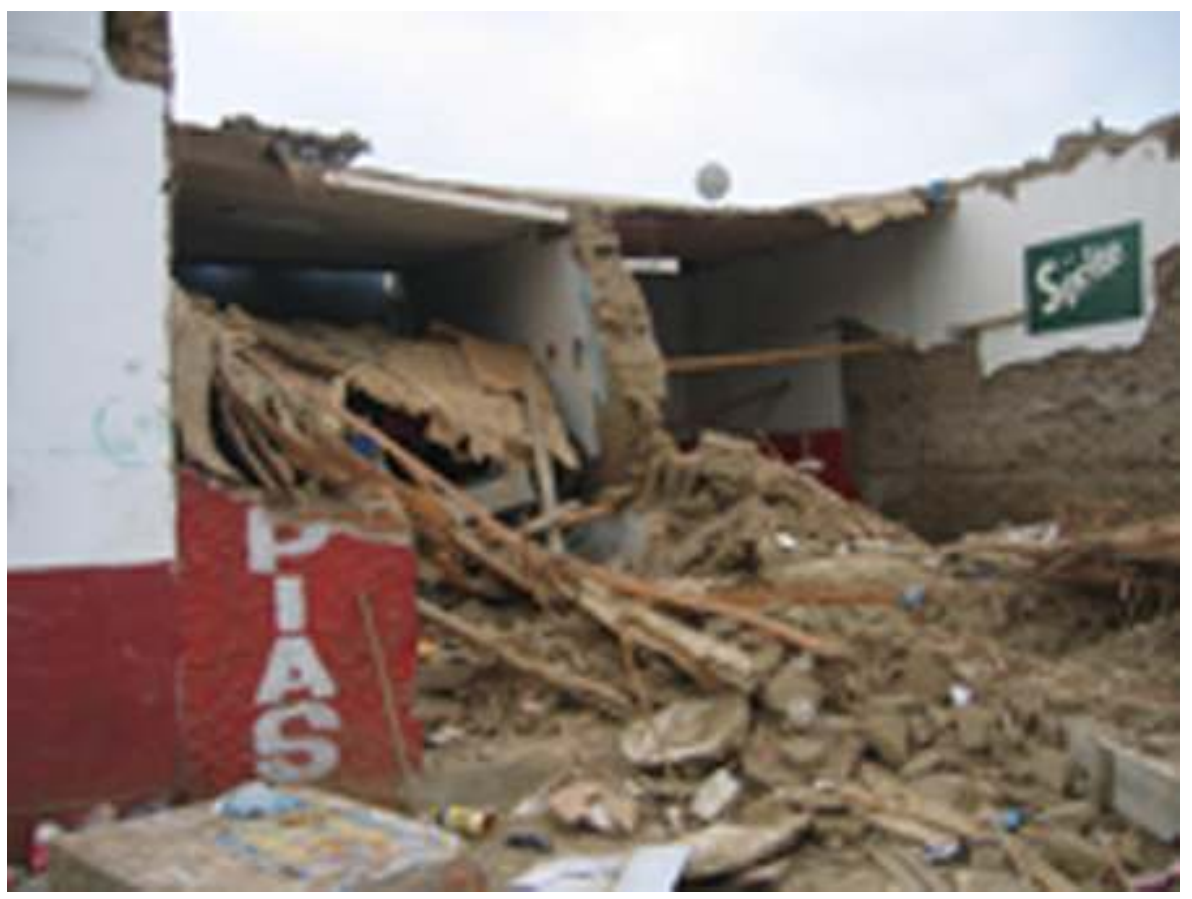

Auteur : Robert D'Ercole - Août 2007

Photo 6 - Dommages à un édifice récent, mal construit (Pisco)

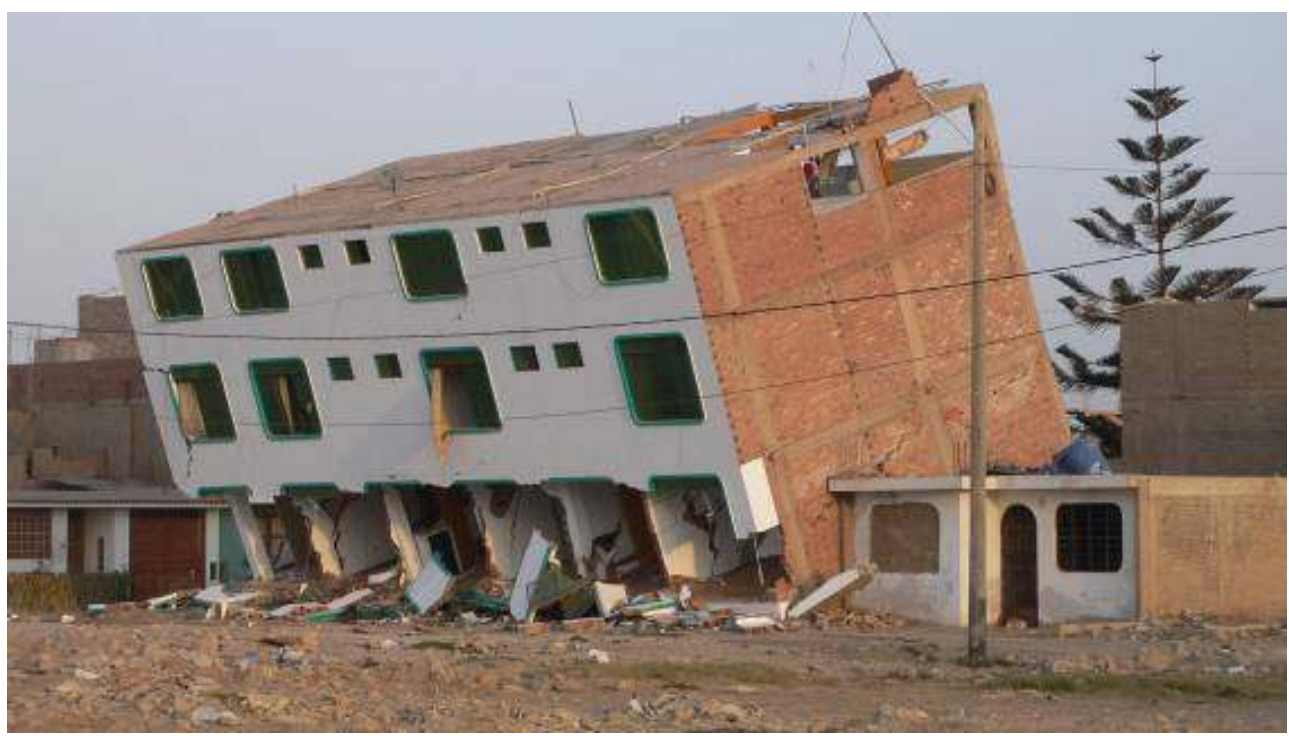

Auteur : Laurence Audin - Août 2007

13 Une répartition sélective des dégâts est notable en fonction du type de construction et du substrat sur lequel les habitations étaient édifiées. C'est ainsi que les constructions en adobe $^{3}$, pour la plupart réalisées de manière informelle, sans aucune norme antisismique ${ }^{4}$ ont été détruites à près de $80 \%$ alors que les constructions en brique ou en béton ont dans l'ensemble mieux résisté. Cependant les dégâts ont concerné tout type de construction lorsque se présentaient des défauts de construction ou lorsque les habitations se trouvaient sur des sols de mauvaise qualité, notamment dans les zones de dépôts 
sédimentaires d'origine marine ou fluviale où la nappe phréatique est peu profonde. Des phénomènes de liquéfaction $\mathrm{du} \mathrm{sol}^{5}$ se sont ainsi produits, déstabilisant et détruisant les habitations comme dans le district de Tambo de Mora où l'ensemble de la communauté (5000 habitants) a été sinistrée. Dans le « vieux » Pisco les dernières constructions de type " colonial» occupées par des institutions publiques du type autorité portuaire ont été détruites.

Photo 7 - Destructions généralisées dans la localité de Tambo de Mora (province de Chincha) principalement liées au phénomène de liquéfaction du sol

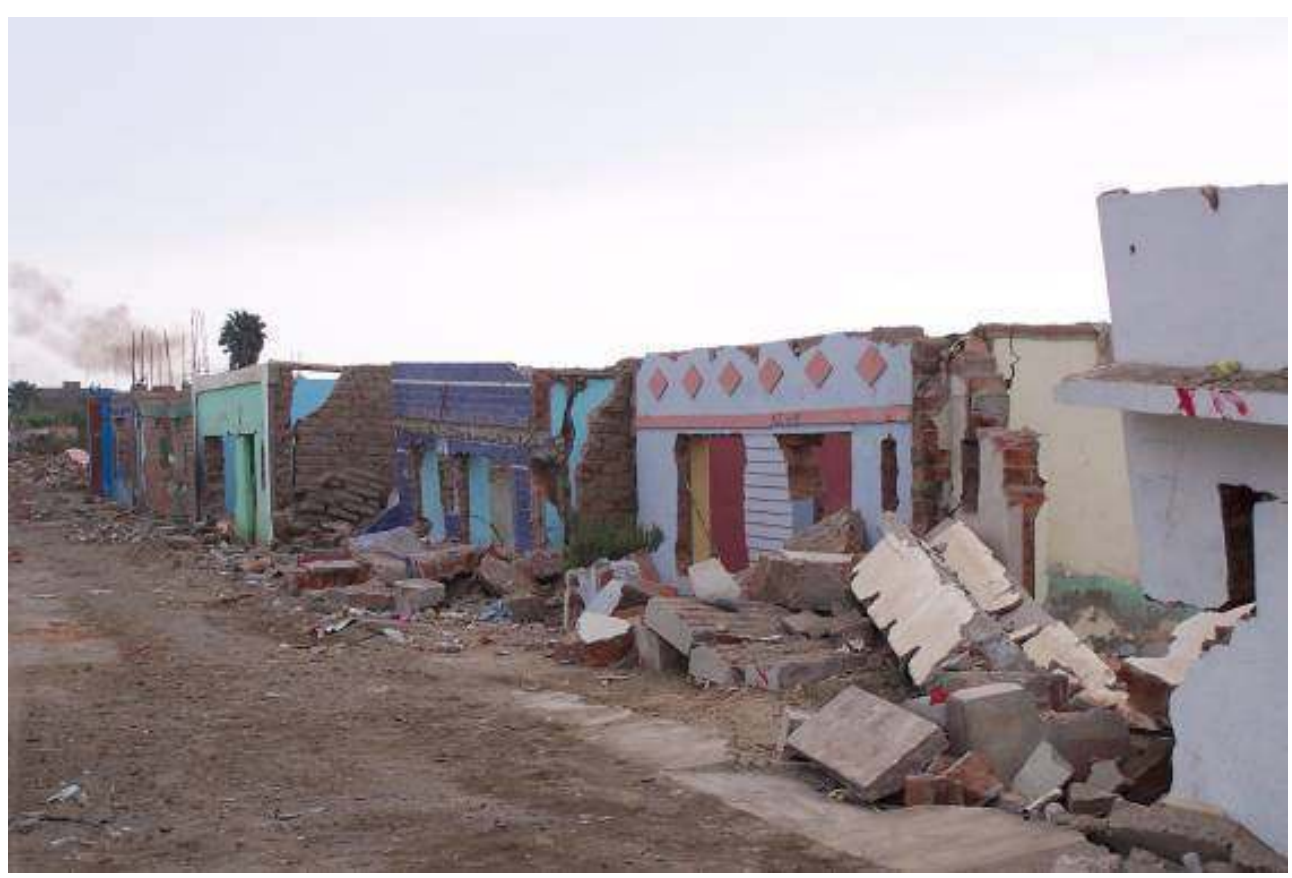

Auteur : Jérôme Chandes - Octobre 2007 
Photo 8 - Effets du phénomène de liquéfaction du sol sur une maison de Tambo de Mora (province de Chincha)

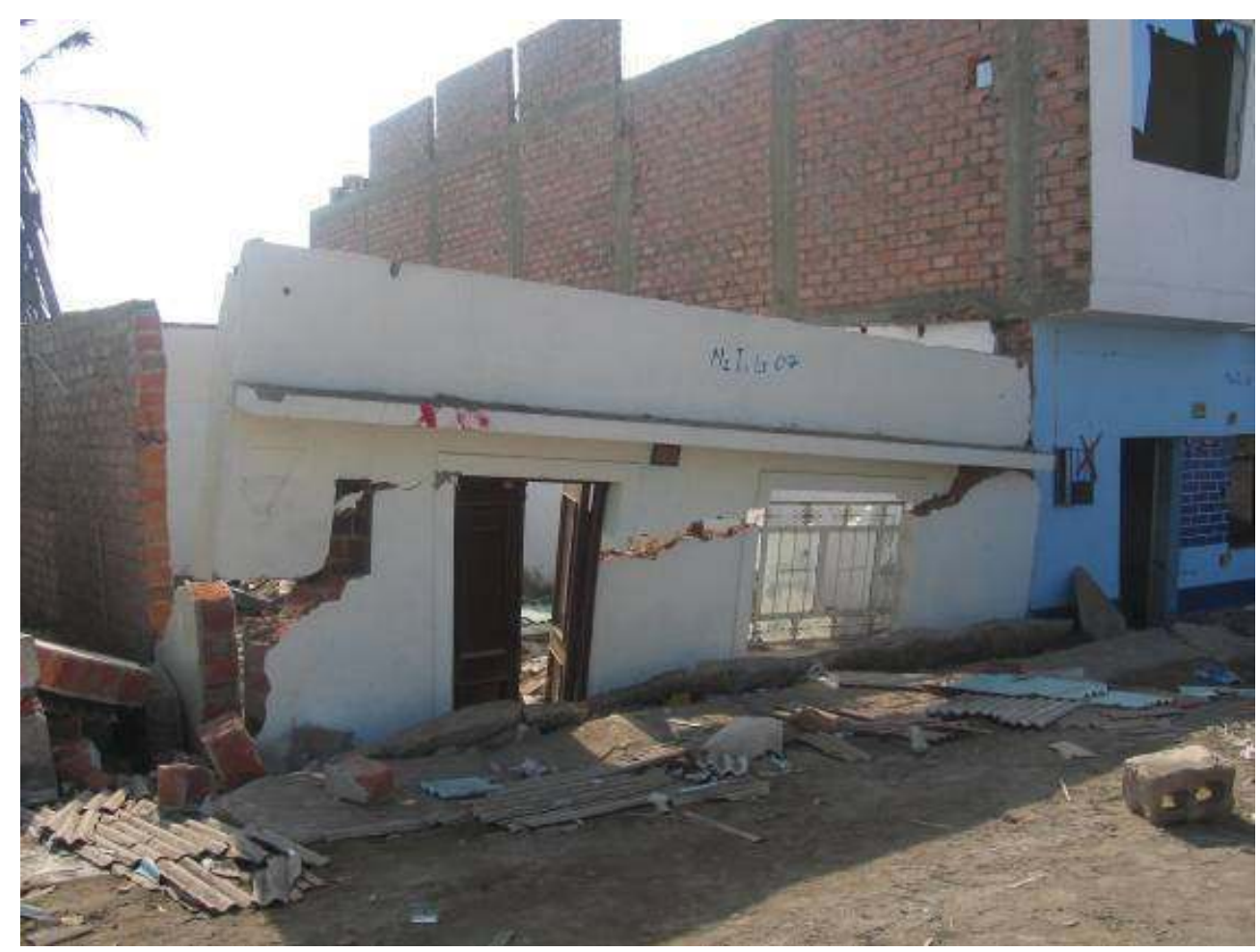

Auteur : Robert D'Ercole - Septembre 2007

\section{Les aléas de la gestion de la crise}

Le séisme du 15 août 2007 a montré les limites de la gestion des crises sismiques au Pérou, en dépit d'un nombre de victimes relativement faible, de la courte distance séparant les zones les plus affectées de la capitale, et de la fréquence de tels événements. Pour les 40 dernières années la base de données du $\mathrm{CRED}^{6}$ reporte 28 catastrophes liées aux tremblements de terre. Le tableau qui suit indique les 7 séismes destructeurs survenus depuis 2001 et leurs principales conséquences.

Séismes destructeurs survenus au Pérou depuis 2001 et principales conséquences

\begin{tabular}{|c|c|c|c|c|c|c|c|}
\hline & Date & $\begin{array}{l}\text { Morts (et } \\
\text { cispasus) }\end{array}$ & Blassés & Sinstrids & Affectes & $\begin{array}{l}\text { Logements } \\
\text { cétruts }\end{array}$ & $\begin{array}{l}\text { Logements } \\
\text { endammagss }\end{array}$ \\
\hline Seisme Araqupa & $2305 / 2001$ & 83 & $2 B 12$ & 215420 & sd & 22052 & 37576 \\
\hline Selisme Capecmarca & $0808 / 2003$ & & & 910 & 4483 & 209 & 1102 \\
\hline Sebisme Moquegua & $2808 / 2000$ & & 11 & 104 & 67 & 2 & 2 \\
\hline Seisme Chuscti & $3006 / 2005$ & & & 1166 & 1486 & 236 & 334 \\
\hline Seisme Moquegua & 01092005 & & & 2789 & 2912 & 10거 & 1876 \\
\hline Soisme San Marth & $26009 / 2000$ & B3] & & 12919 & 19946 & 2य50 & 4204 \\
\hline SAismis PiBco & $15108 / 2007$ & 519 & 1291 & 431813 & 219826 & 92152 & 44810 \\
\hline Total & & 655 & 4114 & 669113 & 243719 & 117607 & 89904 \\
\hline
\end{tabular}

Source : INDECI

15 Suivant la loi du système de protection civile (Ley del Sistema de Defensa Civil) du 29 mars 1972, l'institut National de protection civile, INDECI (Instituto Nacional de Defensa Civil), est responsable des actions à mener durant des situations d'urgence comme celle occasionnée par le séisme du 15 août. Le système de protection civile est normalement décentralisé avec des antennes d'INDECI à l'échelle des régions, provinces et districts. 
L'institution a en fait présenté un double visage: celui, perçu depuis Lima, d'un organisme en mesure de coordonner des opérations d'urgence, et celui, vécu dans les zones sinistrées, d'un organisme bureaucratique, centralisé et doté de peu de moyens, se heurtant à de grandes difficultés pour gérer la crise localement.

En dépit de difficultés de communications entre INDECI et la présidence de la République, l'institution a réagi assez rapidement en envoyant à El Callao (zone portuaire de l'agglomération liménienne) des équipes pour évacuer les familles exposées à un possible tsunami. Le 16 août, d'autres équipes étaient présentes dans la région de Pisco pour tenter d'évaluer les dégâts et les besoins de la population. Avec l'Agence Péruvienne de Coopération Internationale (ACPI), INDECI a organisé, le 17 août, une table ronde réunissant les représentants des milieux diplomatiques étrangers et les organismes de solidarité internationale afin de coordonner l'aide internationale. Dans le même temps INDECI a organisé, avec l'appui des forces armées aériennes, un pont aérien entre l'aéroport militaire de Lima et celui de Pisco, permettant le transport de personnels (équipes médicales, secouristes, militaires...) et de dons composés de produits de première nécessité (eau, alimentation, couvertures, etc.). Ce pont a été maintenu jusqu'au 20 août, le temps de la réhabilitation du réseau routier entre Lima et la zone du sinistre.

Contrastant avec les activités visibles depuis Lima, la gestion locale de la crise a montré les grandes faiblesses du système national de protection civile. Parmi les problèmes les plus graves, figurent :

- la déficience des comités locaux de protection civile, présidés par les principales autorités élues, incapables de s'organiser et d'évaluer les dommages et les besoins des populations sinistrées ;

- le manque d'équipements spécialisés (engins de déblaiement, par exemple) utiles pour les opérations de secours, et pour fournir des refuges aux nombreuses familles ayant perdu leur logement ${ }^{7}$;

- une insuffisante attention aux blessés en raison des graves dommages enregistrés par les établissements de santé ;

- la difficulté d'organiser la distribution de l'aide nationale, et parfois internationale ;

- l'aide d'urgence a principalement concerné les villes, dans un premier temps, notamment la ville de Pisco où les apparitions du président de la République, de ministres péruviens et de personnalités étrangères ont largement été médiatisées alors que les interventions ont tardé dans la province voisine de Chincha, au moins autant affectée, et dans les zones rurales et autres secteurs andins également touchés (Figures 2 et 3$)^{8}$.

Plusieurs semaines ont ainsi été nécessaires pour parvenir à couvrir les besoins de première urgence et pour voir la population sinistrée disposer du minimum nécessaire à sa survie. Une partie d'entre elle a été regroupée dans des camps provisoires, notamment lorsque les destructions étaient généralisées. Dans d'autres cas, des abris provisoires ont été érigés à proximité des habitations détruites, parfois au bord de routes très fréquentées, souvent à proximité de zones de décombres poussiéreuses, à l'origine de problèmes respiratoires. 
Photo 9 - Campement de populations sinistrées sur la place centrale de Tambo de Mora (province de Chincha)

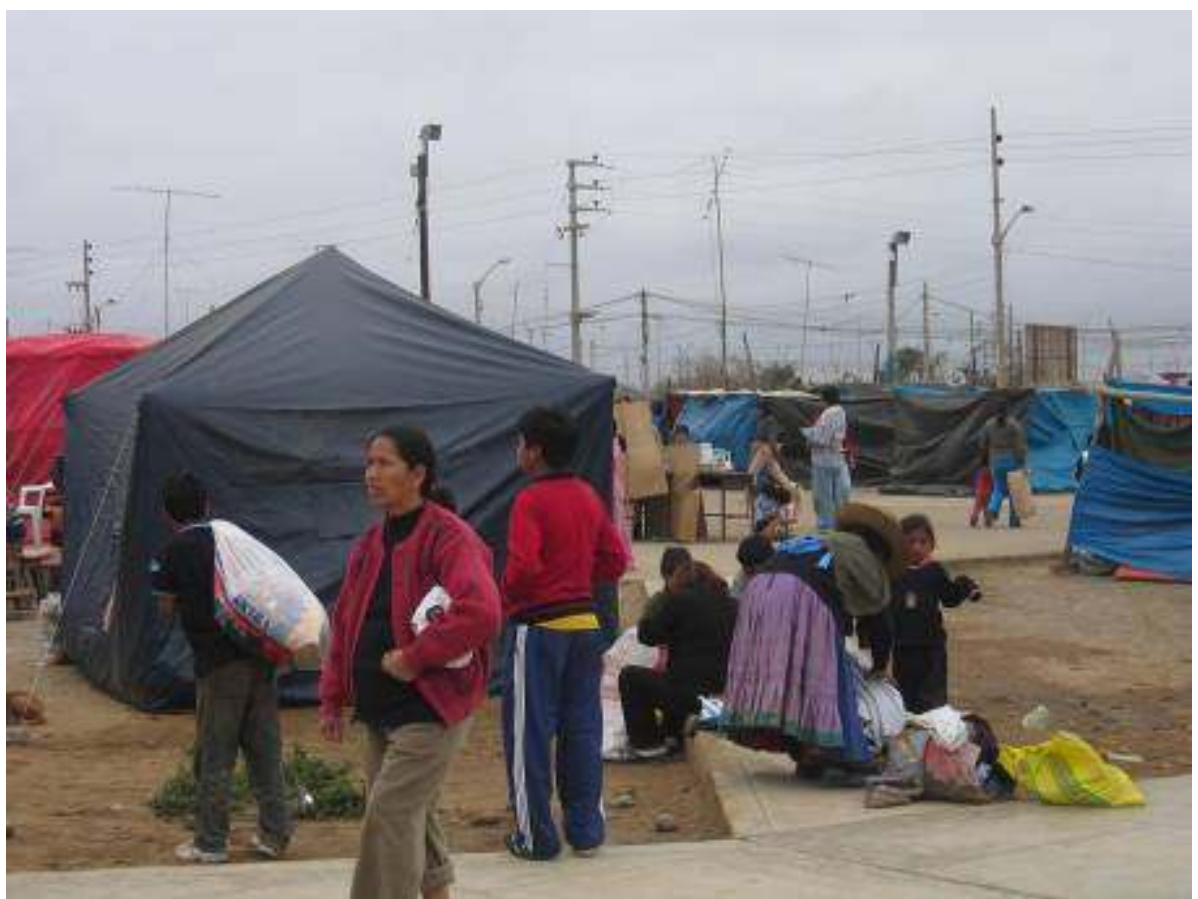

Auteur : Robert D’Ercole - Septembre 2007

Photo 10 - Abris provisoires érigés à proximité immédiate d'habitations détruites (district El Carmen, province de Chincha)

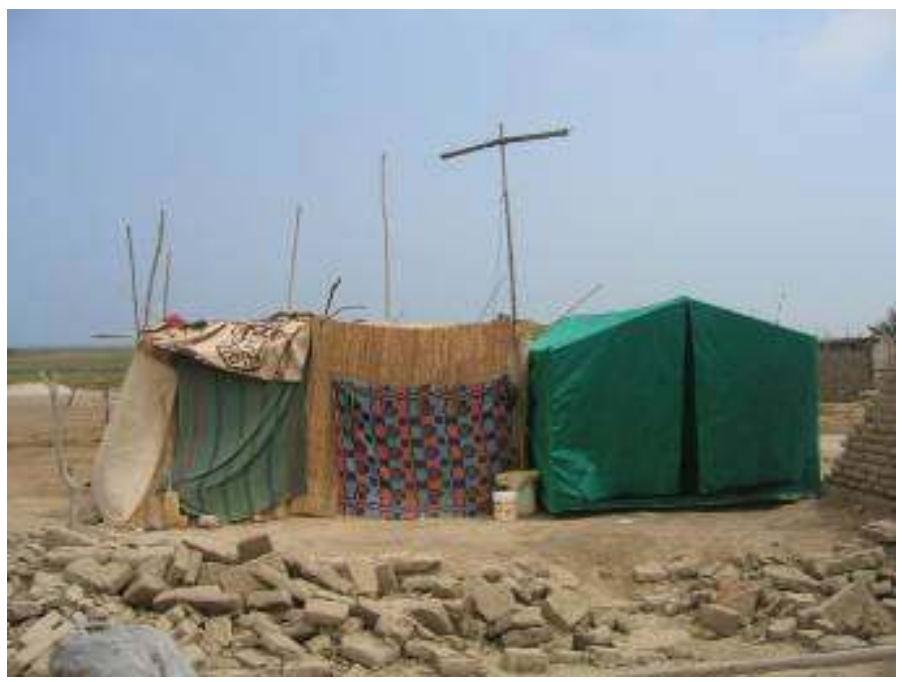

Auteur : Jacopo Zucchelli - Septembre 2007

Dans tous les cas, le souci des familles était de demeurer (du moins un de leurs membres) à proximité du logement détruit ou endommagé. Il s'agissait d'éviter le pillage de ce qui était encore récupérable ou simplement, de contrôler les espaces que ces familles se sont appropriées depuis plusieurs générations. En effet, la plupart d'entre elles ne disposent pas de titres de propriétés et ont donc tout intérêt à contrôler leur territoire. En ce qui concerne les conditions de subsistance, l'approvisionnement alimentaire de la population 
sinistrée a, dans l'ensemble, été assuré, notamment avec l'appui du PMA (Programme Mondial Alimentaire). Des programmes d'urgence, financés notamment par la Communauté Européenne, ont permis d'améliorer les conditions sanitaires et d'alimentation en eau potable, de rendre plus intimes les abris provisoires, d'assurer un minimum de scolarisation aux enfants (généralement sous tente) ou de porter une attention particulière à des personnes handicapées. Ceci dit, les conditions sanitaires et la promiscuité constituent encore des problèmes majeurs.

\section{Entre urgence et reconstruction}

Plus de trois mois après le séisme, il est difficile d'établir une distinction entre l'urgence, la reconstruction et la phase intermédiaire de la réhabilitation des services vitaux. Ces différentes phases que les spécialistes tendent à distinguer dans le temps, se mêlent dans une grande confusion liée à des facteurs aussi divers que le nombre très élevé de sinistrés, les dissensions politiques, la difficile coordination entre les entités nationales et internationales ou encore les temps officiels qui diffèrent de ceux d'une population dont le souhait est de sortir au plus vite d'une situation de grande précarité. Dans certains cas, compte tenu des lenteurs et incertitudes de la reconstruction officielle, la reconstruction informelle a déjà démarré, notamment en milieu rural, avec les pierres effondrées lors du séisme, et dans des conditions qui laissent présager de nouvelles catastrophes dans un futur proche.

21 La reconstruction officielle a pour nom FORSUR (Fondo para la Reconstrucción del Sur). Suivant la loi 29078 du 28 août 2007, cet organisme est chargé du déblaiement des décombres, de la réhabilitation et de la reconstruction «intégrale » des zones affectées par le séisme. Il est dirigé par un entrepreneur, Julio Favre, nommé par Alan Garcia, président de la République. Julio Favre est à la tête d'un directoire réunissant les principales personnalités politiques de la région sinistrée (présidents régionaux, maires provinciaux et de districts) et de quatre représentants du secteur privé. Depuis sa création, FORSUR fait l'objet de vives critiques qui font la une des quotidiens. Outre les opposants politiques d'Alan Garcia, nombreux sont ceux, qui, dans son propre parti (APRA), et dans son administration, contestent le choix d'un représentant du secteur privé, jugeant qu'une reconstruction post-séisme doit être contrôlée par le secteur public et que l'autonomie des élus locaux doit être respectée. Par ailleurs les fonds prévus pour les tâches de FORSUR tardent à venir en raison des blocages bureaucratiques ${ }^{10}$ et de promesses d'aides internationales qui peinent à se concrétiser ${ }^{11}$. Il semblerait que les gouvernements étrangers attendent de voir cesser la polémique qui s'est développée autour de FORSUR et souhaitent des précisions sur les actions concrètes que cet organisme sera amené à réaliser dans divers domaines comme l'alimentation en eau, l'assainissement, le secteur scolaire, hospitalier, le secteur électrique et le logement. En matière de logement, FORSUR promet la construction de milliers de logements (avec des chiffres variables qui restent largement en dessous des besoins), des aides pour leur acquisition provenant de FORSUR ou d'autres programmes nationaux, avec, pour compléter, des prêts bancaires normalement à taux préférentiels ${ }^{12}$.

Pour l'instant cependant, très peu d'activités ont été engagées et les sinistrés sont dans l'impossibilité de planifier leur avenir. Trois mois après le séisme, se pose toujours le problème des décombres, même si, au bout de nombreuses semaines, la plupart des espaces obstrués par les habitations effondrées ont été dégagés, permettant ainsi aux 
familles de déménager leurs abris provisoires et de contrôler leurs propriétés qu'elles soient officielles ou informelles. Ceci dit, les conditions de vie, notamment sur le plan sanitaire, restent déplorables, en dépit des efforts de diverses ONG ou de la Croix Rouge. Par ailleurs, se pose le problème des locataires, relativement nombreux en milieu urbain ${ }^{13}$ , ces derniers, à la différence des propriétaires, pouvant difficilement bénéficier de la reconstruction officielle aussi longue soit-elle à se matérialiser. Les premières opérations de reconstruction viennent cependant de démarrer. En raison des difficultés financières FORSUR a du effectuer des choix. Ils se sont notamment portés sur le secteur scolaire et un simple tirage au sort a permis d'identifier les premiers établissements qui seront détruits, puis reconstruits.

Les tergiversations de l'Etat péruvien face à la reconstruction, l'incertitude et le défaut de confiance vis-à-vis des institutions expliquent que certaines familles n'attendent aucune aide et engagent d'elles-mêmes la reconstruction de leurs logements. Dans certains cas, la situation est particulièrement critique, notamment là où les destructions généralisées ont été principalement liées à la nature marécageuse des sols et aux phénomènes de liquéfaction. Les communautés, comme celles de Tambo de Mora (5000 habitants), dans la province de Chincha, souhaitent se déplacer et occuper des espaces plus sûrs. Les entretiens menés avec le maire de Tambo de Mora et plusieurs membres de la communauté (photo 11) indiquent que des terrains pour reconstruire ont été identifiés mais il s'agit de terrains privés dont le coût dépasse les possibilités de la municipalité. Par ailleurs des études de risques sismiques, à partir de microzonages dans la zone à évacuer et dans les zones possibles d'accueil, sont nécessaires sur le plan légal pour justifier le déplacement collectif et régler les problèmes individuels de propriété du sol. Trois mois après le séisme, de telles études n'ont pas encore pu être réalisées. La population de Tambo de Mora occupe toujours les camps de sinistrés et si l'attente se poursuit, elle n'aura d'autre solution que de reconstruire elle-même, sans technique constructive adéquate et sur des sols particulièrement dangereux. 


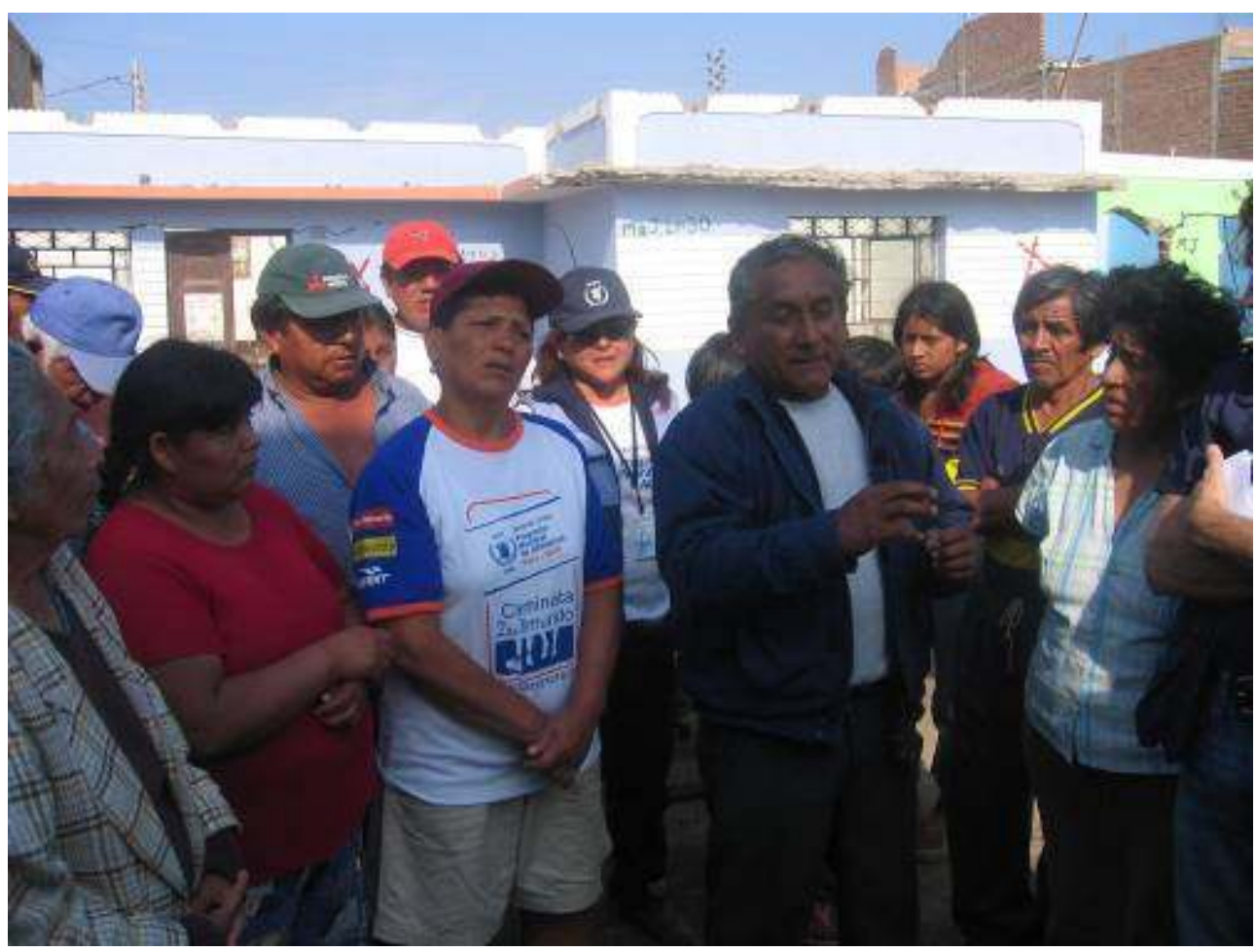

Auteur : Robert D’Ercole - Septembre 2007

\section{Conclusions}

La situation engendrée par le séisme de Pisco du 15 août indique que de toute évidence le Pérou n'a pas su ou n'a pu tirer parti d'expériences similaires, particulièrement nombreuses durant les dernières décennies. Les mêmes causes engendrent les mêmes effets et les causes directes ont pour nom : une forte exposition à l'aléa sismique (parmi d'autres aléas), une absence de planification préventive territoriale, une forte vulnérabilité des populations et de leurs habitations et une capacité de gestion de crise limitée. Depuis le début des années 1990, de nombreuses initiatives internationales (par exemple la Décennie Internationale de Prévention des Catastrophes Naturelles des Nations Unies, 1990-2000), régionales (CAPRADE ${ }^{14}$, PREDECAN ${ }^{15}$, etc.) ou nationales (très nombreux séminaires et ateliers sur la gestion des risques et des crises) ont vu le jour sans pour autant déboucher sur des résultats tangibles en matière de réduction des vulnérabilités et des risques, et d'amélioration des gestions de crises. Cette situation qui n'est pas seulement caractéristique du Pérou, relève de causes de fond qui touchent aux fondements de la société. Le facteur financier est de moins en moins en cause. Le Pérou n'est pas un pays pauvre. Les situations comme celles liées au séisme de Pisco sont la résultante de l'histoire du Pérou (centralisme et bureaucratie notamment), de choix politiques et d'une faible pression sociale, ce qui tend à minimiser la portée d'un risque comme le risque sismique en dépit des dommages matériels et humains considérables qu'il est susceptible d'occasionner.

25 La gestion actuelle de la crise et la reconstruction post-séisme, telle qu'elle est engagée, font présager un accroissement substantiel des vulnérabilités en raison des nombreux 
laissés pour compte pour lesquels le ressentiment vis-à-vis des institutions ne peut que grandir. Il s'agit, en particulier, de ceux qui rebâtiront dans des conditions de grande insécurité compte tenu de la mauvaise qualité des constructions et de la localisation dans des zones à haut risque. S'il fut exceptionnel par ses caractéristiques, le séisme de Pisco ne l'est pas par sa taille. Des séismes beaucoup plus importants peuvent êtres attendus dans la région. Au nord de la dorsale de Nasca, le temps de retour de grands séismes (Mw 8 ou plus) se situe entre 20 et 40 ans. Au sud de cette structure, les séismes sont moins fréquents, avec des temps de récurrence de l'ordre de 120 ans, mais de plus grande magnitude $(M w>8.5)$. La zone du séisme de Pisco avait été identifiée par l'équipe de Hernando Tavera de l'IGP (Institut Géophysique du Pérou) comme une lacune sismique susceptible de générer le séisme observé (Tavera et Bernal, 2005). Ce séisme n'est donc pas une surprise. De telles lacunes existent également face à Lima, et entre Ilo et Arica, au sud du pays. Les séismes correspondants peuvent survenir dans un futur proche et occasionner des dommages humains et matériels très importants. Dans le cas du séisme attendu à Lima, les dégâts devraient être bien supérieurs à ceux de Pisco compte tenu de la concentration humaine que constitue la capitale (8 millions d'habitants), de la médiocre qualité des sols et des constructions, notamment dans les quartiers marginaux ( barriadas) qui regroupent $40 \%$ de la population liménienne.

\section{BIBLIOGRAPHIE}

Audin L., Perfettini H., Bondoux F., Farber D., Cavagnoud R. (2007) - Sud Pérou 23/08/2007 07/09/2007. Rapport de mission d'urgence sur le site d tremblement de terre de Pisco, 15/08/2007 - IRD, Univ. Santa Cruz, IFEA, 62p.

CRDI (1998) - Pérou: habitations en adobe à l'épreuve des séismes - Publications du Centre de Recherche pour le Développement International, http://www.idrc.ca/fr/ev-2689-201-1DO_TOPIC.html

INDECI - Movimiento sísmico afecta severamente departamentos de Ica y Sur de Perú - Informes de emergencia.

Taucer F, Alarcón J., So E. (2007) - 2007 August 15 Magnitude 7.9 Earthquake near the Coast of Central Peru - Report EEFIT (Earthquake Engineering Field Investigation Team), London, 66p.

Tavera H, Bernal I. (2005) - Distribución espacial de áreas de ruptura y lagunas sísmicas en el borde oeste del Perú - IGP, Volumen especial No 6 Alberto Giesecke Mato, Lima, p.89-102.

http://khatati.igp.gob.pe/cns/publi/publi_ult/2005/distribucion_espac_aere_tavera.pdf

Principaux quotidiens et hebdomadaires consultés : El Peruano (notamment pour les textes réglementaires), El Comercio, Peru21, La República, Agenciaperu.com, Carretas. 


\section{NOTES}

1. Ces paramètres sont préliminaires et sont amenés à évoluer, car les définir avec précision nécessite l'incorporation dans les modèles de toutes les données disponibles (sismologiques, géodésiques, observations de terrain, etc.).

2. La destruction des hôpitaux San Juan de Dios et ESSALUD, dans le district de Pisco, a provoqué le décès de 29 personnes et une centaine de blessés. Dans la province d'Ica, les structures de santé Felix Torrealba, Apoyo Santa María del Socorro, Regional et ESSALUD sont à l'origine de la mort de 214 personnes et 487 blessés (INDECI, Informe de Emergencia, n²93, 19/08/2007)

3. L'adobe est une brique de terre crue séchée au soleil. Elle est obtenue à partir d'un mélange d'argile, d'eau et d'un liant utilisé en petite quantité (paille hachée). On estime que $65 \%$ de la population rurale du Pérou, de même que le tiers des citadins, vivent dans des constructions en adobe (CRDI, 1998).

4. Malgré la fragilité de l'adobe, des modes de construction antisismiques existent (ils font l'objet de recherches à l'Université Catholique de Lima) mais sont peu propagés. Les rares habitations construites de cette manière ont correctement résisté au séisme.

5. Selon le BRGM, la liquéfaction est un phénomène qui se produit sous sollicitation sismique provoquant la perte de résistance d'un matériau sableux saturé en eau. La déconsolidation brutale du matériau se traduit par la déstructuration du sol, rendant particulièrement instables les constructions reposant sur ces formations.

6. Le Centre de Recherche sur l'Epidémiologie des Désastres de l'Université de Louvain (Belgique) considère comme catastrophe les événements qui ont répondu à un des critères suivants au moins : 10 morts ; 100 personnes sinistrées et plus; appel à l'aide internationale ; déclaration d'un état d'urgence.

7. L'un des problèmes majeurs fut lié à l'absence de tentes non seulement à l'échelle locale, mais également nationale. Plusieurs semaines furent nécessaires aux principaux intervenants (ONG, organismes internationaux) pour couvrir une partie importante des besoins en abris provisoires (tentes, abris en cannes tressées ou en bois).

8. Ce traitement inégal privilégiant la ville de Pisco ( 60.000 habitants) au détriment d'autres petites villes de moindre réputation, et surtout de zones rurales indiennes ou à forte minorité noire, a largement été commenté localement et accru le ressentiment habituel vis-à-vis des autorités nationales.

9. L'Office Humanitaire de la Communauté Européenne (ECHO) a débloqué 2 millions d'euros pour des actions de première urgence engagées dès le lendemain du séisme et 6 millions d'euros pour des projets d'urgence en continuité avec les précédents.

10. Exemple de la Loi 29136 du 20 septembre 2007 qui modifie la loi 29078 du 28 août 2007 à propos de la création et des fonctions de FORSUR, afin de répondre à certaines critiques formulées à l'encontre de cet organisme.

11. Sur les 100 millions de dollars promis au lendemain de la catastrophe, dont 30 provenant du gouvernement espagnol, seuls 7 millions seraient disponibles au 15 novembre 2007.

12. Ces aides posent problème dans la mesure où l'aide financière promise par le gouvernement à travers FORSUR (6000 soles, soit 2000 dollars américains par logement détruit) est lié aux prêts bancaires. Or, une partie importante des sinistrés, les plus pauvres, n'ont pas accès au système bancaire.

13. Entre 10 et $40 \%$ suivant le lieu, dans la province de Chincha, selon l'ONG italienne COOPI impliquée dans les secours de première urgence.

14. Comité Andin pour la Prévention et la Gestion des Catastrophes. 
15. Prévention des Désastres dans la Communauté Andine: programme européen d'appui institutionnel à la Communauté Andine.

\section{RÉSUMÉS}

Plus de trois mois ont passé depuis le séisme qui, sur la côte péruvienne, à $200 \mathrm{~km}$ au sud de Lima, a tué plus de 500 personnes. Le nombre de victimes est faible au regard des dégâts matériels enregistrés mais le nombre de sinistrés est très élevé. Alors que l'événement est presque oublié à l'échelle internationale, plusieurs dizaines de milliers de personnes vivent toujours dans des conditions précaires dans les abris provisoires fournis par les secours d'urgence, en attendant que ne s'engage le processus de reconstruction. Dans ce contexte, l'objectif de cet article est de procéder à un premier bilan des caractéristiques du séisme, de ses effets différentiels, de la gestion de la crise et de la situation actuelle qui oscille entre urgence et reconstruction.

More than three months have passed since the earthquake, on the Peruvian coast, $200 \mathrm{~km}$ south of Lima, killed more than 500 people. The number of casualties is small in comparison with the damage recorded, but the number of homeless people is very high. While the event is almost forgotten at the international level, tens of thousands of people are still living in precarious conditions in the temporary shelters provided by the emergency, until begins the reconstruction process. In this context, the objective of this article is to make an initial assessment of the characteristics of the earthquake and its effects, the management of the crisis and the current situation that oscillates between relief and reconstruction.

\section{INDEX}

Mots-clés : gestion de crise, Pérou, Pisco, reconstruction, séisme

Keywords : crisis management, earthquake, Peru, Pisco, reconstruction

\section{AUTEURS}

\section{ROBERT D'ERCOLE}

Robert d'Ercole (robert.dercole@ird.fr) est géographe à l'IRD, Lima, Pérou.

\section{JÉRÔME CHANDES}

Jerôme Chandes (jchandes@yahoo.com) est doctorant « logistique et stratégies » au CRETLOG, Université de la Méditerranée, Aix-en-Provence.

\section{HUGO PERFETTINI}

Hugo Perfettini (perfetti@lmtg.obs-mip.fr) est géophysicien à l’IRD, Lima, Pérou.

\section{LAURENCE AUDIN}

Laurence Audin (laurence.audin@ird.fr) est sismotectonicienne à l'IRD, Lima, Pérou. 